\title{
Apps that help the elderly with dementia
}

\author{
Adriana Kozina, Valentina Rok, Andrej Starc \\ University of Ljubljana, Faculty of Health Sciences, Ljubljana, Slovenia. \\ E-mail:adri.kozina@gmail.com; ms.valentina.rok@gmail.com; andrej.starc@zf.uni-lj.si
}

\begin{abstract}
Introduction: In the year 2018, it was estimated that at the global level were 50 million patients diagnosed with dementia, and the cost of their treatment had risen to over one billion US dollars. With the growth of the elderly population, that number shall only grow higher in the future. Research shows that specific mobile apps on smart devices could be used to help patients with dementia. The usage could reduce the financial cost of this illness, also. On the other hand elderly people do not have sufficient knowledge of the apps, and dementia influences their cognitive functions. Methods: We used a descriptive method with critical analysis of English scientific literature, using databases Cochrane Library, Google Scholar, PubMed, ScienceDirect and Wiley Online Library. The keywords used were: apps for dementia, apps for elderly with dementia, apps for dementia prevention. Between the words was used the Boolean logical operator AND. We included the literature published between 2013 and 2020, which were free and fully accessible. Results: We detected 1140 articles after the inclusion of all criteria. Only 8 scientific papers met the inclusion criteria and were about apps for patients with dementia. In the smart mobile marketplace exist several mobile apps, which are used for: diagnosing people with dementia, prevention for developing dementia, enhancing cognitive functions and apps for helping patients with everyday activities. Most free and accessible apps do not cover all the specific needs of people diagnosed with dementia. We expect that soon, there shall be a rise in such mobile apps development. Discussion and conclusions: Based on the appropriate design and the technology, app's could be used for improvement of physical, mental and social aspects of persons diagnosed with dementia. Mobile apps used for preventive strengthening of cognitive functions or for control and slowing the progress of dementia have proved to be an adequate tool for quick and efficient assessment of dementia. The development of such
\end{abstract}


apps is requires inclusion of healthcare workers as well as technological scientists and patients. More research should be done concerning app efficiency and adjustments of their functions for the specific patients' needs.

Keywords: Apps for dementia, elderly with dementia, apps for elderly with dementia

\section{Introduction}

Dementia is a syndrome for which it is typical a progressive lessening of the cognitive and behavioural abilities and memory and aggravation of doing everyday activities, which usually afflicts the elderly. In 2018, there was an estimation that around the world, there were 50 million people with dementia and treatment costs were a billion American dollars; however, the number of afflicted will increase till the year 2050 for a third (ADI - Alzheimer's Disease International, 2018). Research shows that specific mobile apps, meant for people with dementia and their caretakers, could lower their physical and psychological burden. At the same time, they could also reduce the costs to the Healthcare systems, higher the chances of the patients' independence and acceptance of their chronic disease while also slower its' development (Klimova, 2017). On the market, we can find a variety of apps. They can work preventively, meaning that they can serve as a diagnostic tool or quicker detection of developing dementia (Klimova, 2017).

On the other hand, apps can also work as a helping tool, making patients' lives more manageable and help them to cope in their everyday life (Klimova, 2017). Mobile apps could be beneficial at controlling dementia in five areas. Firstly, on detection and assessment of dementia disease, secondly at cognitive training of people with this diagnosis, thirdly for the evaluation of health and safety of people with dementia, fourthly in socialisation and relaxation and fifthly in long-distance health assessment of patients by a health professional (Yousaf et al., 2019)

It is necessary to know the limitation of usage of apps by the elderly since they are known to have limited knowledge of using technology, and dementia is known to cause further cognitive decline. Consequently, the usage of apps must be adjusted to the users - they need to be simple to use and calculate their needs and disadvantages. It is essential to add that all apps are not made by experts and are, therefore not adequate for use (Choi, 2020).

\section{Methods}

In this research it was used the descriptive method with critical appraisal of English scientific literature with the assistance of the diKUL portal (Digital library of University of Ljubljana). We used next keywords with Boolean logical operator AND: Apps for dementia, elderly with dementia, apps for elderly with dementia. The included literature needed to correspond with the following requirements: published between the years 2013 and 2021, the paper required to 
be recessed, be in full text, had open access and be written in either English or Slovenian language. On Figure 1 it is shown the process of selecting suitable articles.

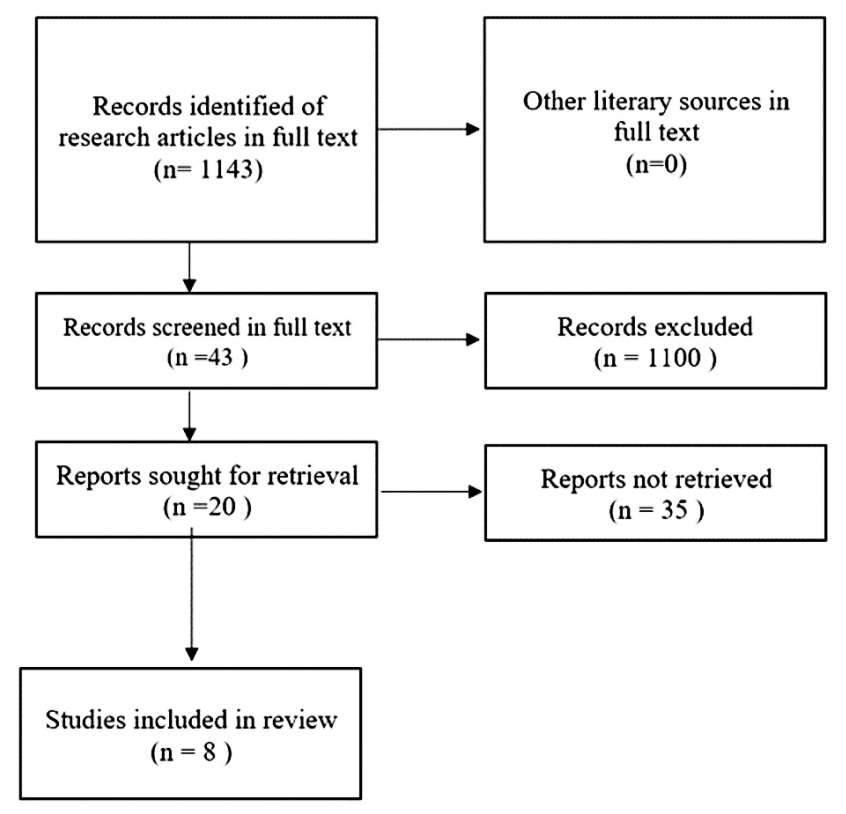

Figure 1: PRISMA diagram

\section{Results}

In Table 1, there are descriptive results of the studies that were included in this research. What we found noteworthy is the similarity between the results of these eight studies.

\section{Table 1: Results of literature review}

\section{Authors} ical applications in the field of

Identify key areas of technological develop-

Astell et al., 2019 ment in the field of dementia treatment and propose guidelines for future work
Overlook of recent research findings. dementia are:

a) diagnosis, assessment and monitoring,

b) maintenance of operation,

c) leisure and activities,

d) care and management. A holistic approach to the problem is needed. 
9 studies were included. Tech-

Review the findings of

Brown, O'Connor, 2020. recent qualitative studies to date on the use of mobile applications for peoQualitative literature overview. nology can improve some aspects of the physical, mental and social health of people with dementia. It can serve to promote cognitive functions and communication skills in people with dementia.

Applications differ from each other in quality and usability. $41.7 \%$ of the examined applications were not developed by healthcare professionals and were of lower quality information compared to the applica-

Overview and evaluation tions developed by healthcare of the features, content and technical aspects of 36 applications aimed at Google Play and the App store in the USA. Applications were coded and rated using a mobile app rating scale. plications for people with dementia. More than $50 \%$ of the
Review existing applications that contain information on Alzheimer's disease and other dementias and assess their usefulness professionals. This suggests the need for collaboration between healthcare professionals and IT professionals in developing apapplications were aimed at caregivers of people with dementia. Only one-third of the applications contain a reminder function for patients or caregivers. Applied with a proper design, applications can go a long way in making dementia management easier.

Currently, accessible apps do not cover all the complex needs of people with dementia and can be difficult for patients to use due to reduced abilities as a result of the disease. The authors emphasize that it would be necessary to develop properly designed, high-quality applications that could make it easier for patients to cope with the disease and help them to be more independent in managing the disease, while facilitating the work of their caregivers and families.

Studies have shown that mobile apps can be an excellent tool for quickly and accurately assessing dementia. They also reduce the psychological and financial burden on patients and their carers. More research should be done on the effectiveness of applications and, in collaboration with designers, their functions should be adapted to the specific needs of people with cognitive deficits. 
First study: The most frequent-

The first study identified important activities for people with dementia in the past and present, and the second study explored the needs, wants, and capabilities of us-

Kerkhof, 2017 ing mobile apps to help manage dementia. Findings could help to develop a tool to facilitate selecting suitable applications for the individual according to his abilities and needs. ly mentioned meaningful activity was 'being socially active'. Researchers have also found that an individual's activities before dementia carry essential information about important activities to the present. The most frequently mentioned needs for disease self-management by sufferers related to the support of memory functions. In another study, the authors detected some wishes of patients that had not previously been seen in the literature - minimal need to type to use applications, the ability to adjust the language, the ability to rotate the displayed image.

The first study implements a systematic and methodological approach in the development of a particular application. The created application has been well received by users and needs some updates and customisation of certain features.
A systematic approach to mobile application development.
The CAIDE Risk score app is the first app to predict the risk of dementia with the help of an evidence-based tool. It can be used by healthcare professionals in their work or by individuals suspected of having dementia. was developed based on the CAIDE Dementia

Risk scale. scale accessible to all users who want to determine whether they have dementia.

\section{Discussion}

Mobile apps have become quite accessible to users of mobile phones and tablets these days. The number of the latter is multiplying. Based on this, we can conclude that, as a result, new possibilities are opening up for reaching people with dementia and for preventive action in this area. Based on current studies, we can conclude that appropriately designed mobile apps can be an effective tool for dementia management (Klimova, 2017; Choi, 2020). The use of apps is possible in different areas. The researchers classified technological applications into four main groups, according to their purpose or content: apps that serve to facilitate the diagnosis, assessment and monitoring of patients, applications that support their operation, applications for activities and leisure and apps for care and disease management (Astell et al., 2021). The main positive effects of the applications identified by various researchers are reduction of the psychological and financial burden on patients and carers, the reduced financial burden of disease for the health system, rapid diagnosis and assessment of 
condition, assistance to patients and carers in everyday life, maintenance of social contacts and maintenance cognitive abilities - cognitive training (Klimova, 2017; Yousaf et al., 2020; Cindy et al., 2015; Rai et al., 2020a).

Due to the nature of the disease, applications must also be adapted accordingly. Studies have shown that many apps are not created in collaboration with health professionals and people with dementia. The latter announces questionable quality. Some do not have good information value, do not cover the specific needs of users, and do not take into account cognitive decline (Rai et al., 2020b; Klimova, 2017; Kerkhof et al., 2017).

There are many apps available to users, which differ in quality. Each also contains only certain content and functions, which may mean that patients would need an even more significant number of different apps to cover all their needs. To date, no apps have been developed that would be well-suited for people with dementia and would include all the necessary features and information. Researchers warn that adequately designed, high-quality apps should be developed to make it easier for patients to cope with the disease in the future. This will help them become more independent in managing the disease, while making it easier for their carers and family (Guo et al., 2020).

\section{Conclusion}

Research has shown that appropriately designed apps correlated with intelligent technology can be used as a preventive tool in dementia management and aid for people who already have a dementia. Currently, no quality apps have been developed that would be adapted to such people's complex needs. We believe that collaboration between healthcare professionals, technology professionals and patients is key to producing such apps. Further research should be done on the importance and effectiveness of apps, and their functions should be adapted to the specific needs of older people with cognitive deficits.

\section{References}

ALZHEIMER'S DISEASE INTERNATIONAL, 2018. World Alzheimer Report 2018 The state of the art of dementia research: New frontiers. London: Alzheimer's Disease International. Available at: https://www.alzint.org/ resource/world-alzheimer-report-2018/

ASTELL, A., BOURANIS, N., HOEY J et al., 2019. Technology and dementia. The future is now. Dementia and geriatric cognitive disorders, vol. 47, pp. 131-139. doi: 10.1159/000497800 .

BROWN, A. and O'CONNOR, S., 2020. Mobile health applications for people with dementia. A systematic review and synthesis of qualitative studies. Informatics for health and social care, vol. 45, no. 4, pp. 343-59. doi: 10.1080/17538157.2020.1728536 . 
CHOI, S.K., YELTON, B., EZEANYA, V.K., KANNALEY, K., FRIEDMAN, D.B., 2018. Review of the Content and Quality of Mobile Applications About Alzheimer's Disease and Related Dementias. Journal of applied gerontology, vol. 39, no.6, pp: 601-608. doi: 10.1177/0733464818790187.

GUO, Y., YANG, F., HU, F., LI, W., RUGGIANO, N., LEE, H.Y., 2020. Correction: Existing Mobile Phone Apps for Self-Care Management of People With Alzheimer Disease and Related Dementias: Systematic Analysis. Journal of medical internet research ageing, vol. 3, no. 1, e18754. doi: 10.2196/18754.

KERKHOF, Y., BERGSMA, A., GRAFF, M., DRÖES, R., 2017. Selecting apps for people with mild dementia: Identifying user requirements for apps enabling meaningful activities and self-management. Journal of rehabilitation and assistive technologies engineering, no. 4. doi: $10.1177 / 2055668317710593$.

KLIMOVA, B., 2017. Mobile phone apps in the management and assessment of mild cognitive impairment and/or mild-to-moderate dementia: An opinion article on recent findings. Frontiers in neuroscience, no. 11, pp: 461. doi: 10.3389/fnhum.2017.00461.

RAI, K.H., SCHNEIDER, J., ORRELL, M., 2020 a. An individual cognitive stimulation therapy app for people with dementia. Development and usability study of thinkability. Journal of medical internet research ageing, vol. 3, no. 2. doi: 10.2196/17105 .

RAI, K.H., CAVALCANTI BARROSO, A., YATES, L., SCHNEIDER, J., ORRELL, M. 2020 b. Involvement of People With Dementia in the Development of Technology-Based Interventions: Narrative Synthesis Review and Best Practice Guidelines. Journal of medical internet research, vol. 22, no. 12, e17531. doi: 10.2196/17531.

SINDI, S., et al., 2015. The CAIDE Dementia Risk Score App. The development of an evidence-based mobile application to predict the risk of dementia. Alzheimer's \& Dementia: diagnosis, assessment \& disease monitoring, no. 1, pp: 328-333. doi: 10.1016/j.dadm.2015.06.005.

YOUSAF, K., MEHMOOD, Z., SABA, T., REHMAN, A., MUNSHI, A., ALHARBEY, R., RASHID, M., 2019. Mobile-Health Applications for the Efficient Delivery of Health Care Facility to People with Dementia (PwD) and Support to Their Carers: A Survey. BioMed research international, vol. 2019, 1-27. doi: 10.1155/2019/7151475. 